TRABAJOS ORIGINALES

\title{
Efecto de atributos paisajísticos en los patrones de presencia de Ateles fusciceps en el noroccidente ecuatoriano
}

\section{Landscape attributes effects on Ateles fusciceps presence patterns in northwestern Ecuador}

\section{Pablo Medrano-Vizcaíno}

Facultad de Ciencias Biológicas, Universidad Central del Ecuador, Av. Yaguachi y Numa Pompilio, Quito, Ecuador. Centro de Biología, Laboratorio de Zoología, Universidad Central del Ecuador, Av. Gato Sobral y Jerónimo Leiton, Quito, Ecuador. Email Pablo Medrano: pabmedrano@hotmail.com

\begin{abstract}
Resumen
Este trabajo evalúa el efecto que tienen ciertas variables paisajísticas (ríos, tierras agropecuarias, áreas antrópicas y bosque nativo) en los patrones de presencia del mono araña, Ateles fusciceps, en el noroccidente ecuatoriano. Se utilizaron registros geográficos de Ateles fusciceps tomados en campo y de estudios previos. Se evaluó el efecto de la proximidad de cada variable por medio de la prueba T de Student. Posteriormente mediante regresiones logísticas y por medio del Criterio de Información de Akaike (AIC) se seleccionaron los mejores modelos y se identificaron las variables más importantes. Se observó que tierras agropecuarias y zonas antrópicas tienen un efecto negativo para este primate, pues los puntos de presencia se encontraron alejados de éstas. Se evidencia también que Ateles fusciceps prefiere sitios cercanos a bosque, resultado que corrobora investigaciones previas, sin embargo también se encuentra una asociación con ríos, resultado que no ha sido reportado en estudios anteriores. Se encontraron dos modelos importantes para predecir patrones de presencia de este primate, el primero compuesto por: bosque nativo, ríos y zonas antrópicas ( $\mathrm{AlCW}=0.48$ ), mientras que el segundo abarca: bosque nativo, ríos, zonas antrópicas y tierras agropecuarias ( $\mathrm{AlCw}=0.34$ ). Estos resultados servirán de base para futuros análisis, dirigidos a la conservación de A. fusciceps.
\end{abstract}

Palabras clave: ecología del paisaje; primatología; mono araña; AIC; regresiones logísticas.

\section{Abstract}

In this work, I investigate the effect of some landscape variables (rivers, agricultural lands, anthropic areas and native forest) on the presence patterns of spider monkey, Ateles fusciceps, in the northwestern Ecuador. Geographical records collected in field and others from previous studies were used to conduct this study. Effects of proximity of each variable to presence of $A$. fusciceps were assessed with Student $T$ tests. The best model and the most important variables were identified using logistic regressions and the Akaike Information Criterion (AIC). The results showed that agricultural lands and anthropic zones were from the primate presence points, suggesting a negative effect on $A$. fusciceps. I also found that $A$. fusciceps prefers sites near rivers, this observation has not been reported in previous studies. Two important models were found to predict the presence of $A$. fusciceps, the first one was composed by three variables: native forest, rivers and anthropic areas $(\mathrm{AICW}=0.48)$ and the second model was composed by the four variables: native forest, rivers, anthropic areas and agricultural lands $(\mathrm{AlCW}=0.34)$. The results of this work will contribute a basis for future studies aimed on $A$. fusciceps conservation.

Keywords: landscape ecology; primatology; spider monkey; AIC; logistic regressions.

\section{Citación:}

Medrano-Vizcaíno P. 2018. Efecto de atributos paisajísticos en los patrones de presencia de Ateles fusciceps en el noroccidente ecuatoriano. Revista peruana de biología 25(3): 241 - 248 (Agosto 2018). doi: http:// dx.doi.org/10.15381/rpb.v25i3.13548

$\begin{array}{ll}\text { Presentado: } & 25 / 07 / 2017 \\ \text { Aceptado: } & 10 / 07 / 2018 \\ \text { Publicado online: } & 25 / 09 / 2018\end{array}$




\section{Introducción}

El análisis y estudio espacial se constituye como una herramienta útil para valorar la situación de los paisajes y realizar interpretaciones morfológicas y funcionales de un territorio por medio de patrones temporales y espaciales. Considerando que los diferentes componentes físicos y biológicos están relacionados y conectados por interacciones dinámicas (Forman \& Godron 1986), resulta relevante el entendimiento de la configuración espacial de las áreas para entender sus procesos ecológicos (Turner 2005).

En lo que respecta a las relaciones entre elementos del paisaje, se deben considerar aspectos como el desarrollo y dinámica de la heterogeneidad espacial, las interacciones a lo largo de paisajes heterogéneos y la influencia de la heterogeneidad espacial en los procesos bióticos y abióticos (Risser et al. 1984). Altos niveles de heterogeneidad paisajística pueden estar relacionados con un elevado grado de fragmentación de hábitats, siendo esto evidente a través de aspectos como la disminución del hábitat, la disminución del tamańo de fragmentos, aumento del número de fragmentos y la mayor distancia entre fragmentos (Saunders et al. 1991, Andrén 1994, Fahrig 2003).

Los primates son uno de los grupos más afectados por los procesos de heterogeneidad paisajística, siendo la fragmentación de hábitat una de las principales causas para el declive de sus poblaciones (Mittermeier et al. 2005). Por otra parte, aspectos como la disponibilidad de alimento, ubicación de cuerpos de agua y variación del riesgo de predación en diferentes hábitats, juegan un rol importante en su distribución (Cowlishaw 1997, Altmann \& Altmann 1970, Zhang \& Wang 1995, Agetsuma \& Noma 1995, Defler 1996, Olupot et al. 1997), siendo evidente la importancia del análisis del paisaje asociado a cada especie para comprender el efecto que tienen las características paisajísticas en los patrones de presencia de los organismos.

En Sudamérica se reportan algunos estudios que analizan la relación entre atributos paisajísticos y la presencia de algunas especies. En el norte de Manaos-Brasil, se observó que el tamaño de parche de bosque y la distancia al área boscosa más cercana, está asociada con la presencia de seis especies de primates, entre ellas una del género Ateles (Boyle \& Smith 2010). En Ecuador, Spaan et al. (2013), determinaron que la densidad de árboles y la altitud son indicadores significativos para la presencia de esta especie. A pesar de lo encontrado, existen otras variables paisajísticas que podrían ser importantes.

En Ecuador habita la subespecie Ateles fusciceps fusciceps, la cual se encuentra en bosques húmedos tropicales y subtropicales de la costa norte y centro entre los 100 y $1700 \mathrm{~m}$. Se distribuye principalmente en la provincia de Esmeraldas, pero también está en el norte de Manabí y en las estribaciones occidentales de Imbabura y Pichincha (Mittermeier et al. 2005, Boada 2014).

La poca información que existe sobre requerimientos paisajísticos para la ocupación de determinados tipos de hábitats, se constituye en una gran limitante para proponer medidas de manejo adecuadas que permitan la conservación de este primate que se encuentra en peligro crítico, debido a factores como la cacería, deforestación y la expansión de la frontera agrícola (Cuarón et al. 2008, Mittermeier et al. 2012).

El principal objetivo de este estudio es analizar la influencia que tienen ciertos atributos del paisaje (ríos, zonas antrópicas, bosque nativo y tierras agropecuarias) en la presencia de Ateles fusciceps en el Noroccidente del Ecuador, a escala de paisaje. Además, se identifican los modelos y variables paisajísticas más importantes para la presencia de Ateles fusciceps y se evalúa la influencia de la proximidad de las variables antes indicadas en la presencia de la especie.

\section{Material y métodos}

Área de estudio.- Se usaron registros geográficos de la presencia de Ateles fusciceps en las provincias de Esmeraldas, Imbabura y Pichincha. Estas provincias se ubican en el noroccidente ecuatoriano y forman parte de El Chocó, región conocida por ser un hotspot de diversidad biológica a nivel mundial, pero, a pesar de su importancia ecológica, está muy afectada por la degradación ambiental (deforestación y expansión de frontera agrícola) y tasas elevadas de extinción de especies (Myers et al. 2000, Dodson \& Gentry 1993).

Población.- Tomando en cuenta que con el pasar del tiempo podrían haber existido cambios en el uso de suelo, se consideraron los registros geográficos más actuales posibles. El registro más antiguo tiene seis años de diferencia con respecto a los datos de las capas en Sistemas de Información Geográfica (SIG) usadas. Se trabajó con 52 registros geográficos de Ateles fusciceps provenientes de estudios previos, cuyos registros fueron colectados entre los ańos 2007 y 2011 y de trabajo de campo realizado durante los meses de noviembre y diciembre del 2015.

Métodos.- Para los análisis espaciales se usaron las capas vectoriales en SIG de ríos a una escala 1:250.000 (IGM 2013) y de cobertura y uso de la tierra del Ecuador continental 2013 - 2014 a escala 1:100.000 (MAE-MAGAP, 2015). Para el trabajo de campo se tomó la localización geográfica de los grupos observados con un Sistema de Posicionamiento Global (GPS) Garmin en coordenadas UTM Datum WGS 84.

Se evaluaron los patrones de presencia de Ateles fusciceps en función de atributos como: a) distancia a bosque nativo más cercano, b) distancia a río más cercano, c) distancia a área antrópica más cercana y d) distancia a tierras agropecuarias más cercanas. El área de corte para el análisis espacial abarcó Esmeraldas, que es la provincia donde se encontró la mayor cantidad de registros geográficos y zonas de las provincias de Imbabura y Pichincha donde se reporta la presencia de este primate (Fig. 1).

Análisis Estadístico.- Como análisis preliminar, se realizó una matriz de correlaciones de Pearson para medir el grado de relación entre las variables predictivas utilizadas y de esta manera determinar si existe dependencia entre éstas y establecer la manera en que se agrupen los datos para su posterior análisis. Posteriormente, para evaluar la relación del paisaje en la presencia de Ateles fusciceps, se realizaron los siguientes análisis:

- Para conocer el efecto que tiene la proximidad de los atributos paisajísticos en la distribución de Ateles fusciceps, se compararon distancias a las variables entre los 52 sitios de presencia y 52 pseudoausencias creadas por medio de puntos aleatorios. Este análisis se realizó mediante una prueba T de Student con un valor $\mathrm{Q}=0.05$.

- Para conocer la relación entre los patrones de presencia de Ateles fusciceps con los atributos paisajísticos se utilizaron 104 registros geográficos, 52 puntos de presencia y 52 pseudoau- 
sencias. Los puntos al azar fueron asociados con las mismas variables predictivas que los puntos de presencia (distancia a bosque nativo más cercano, distancia a río más cercano, distancia a área antrópica más cercana y distancia a tierras agropecuarias más cercanas). Se aplicaron regresiones logísticas con todas las combinaciones posibles de las variables predictivas (quince modelos). Estos análisis fueron efectuados con el Software SPSS (2009). Posteriormente se seleccionó el mejor modelo por medio del Criterio de Información de Akaike (AIC) y se identificaron las variables más importantes por medio de la sumatoria de los pesos de AIC $\left(\mathrm{AIC}_{\mathrm{w}}\right.$ ) de cada variable en los modelos donde fueron incorporadas.

El criterio de información de Akaike (AIC) es un método que permite seleccionar entre un grupo de modelos, el o los modelos que mejor se ajustan a los datos, siendo el mejor aquel que presenta el menor valor AIC. La ecuación que define este criterio es:

$$
A I C=-2 \log (L)+2 k
$$

Dónde $\mathrm{k}$ es el número de parámetros y L la verosimilitud del modelo. Para facilitar la selección de los modelos más importantes se calculan los pesos de $\mathrm{AIC}\left(\mathrm{AIC}_{\mathrm{w}}\right)$, para lo cual primero se obtienen los valores de Delta AIC $(\triangle \mathrm{AIC})$ :

$$
\Delta_{\text {AIC }}=\mathrm{AIC}_{\mathrm{x}}-\operatorname{minAIC}
$$

Dónde, $\mathrm{AIC}_{\mathrm{x}}$ es el AIC del modelo y minAIC es el AIC más bajo o "mejor modelo". Posteriormente se ajustan los datos a una escala de 1 (Akaike 1992, Burnham \& Anderson 2002, Mazerolle 2006).

\section{Resultados}

Análisis de correlación de variables predictivas.- La correlación entre las variables predictivas no es significativa (Tabla 1), con lo que se constata que cada una de las variables predictivas es independiente entre sí y que el agrupamiento de datos para el análisis estadístico debe realizarse de la misma manera.

Tabla 1. Matriz de correlación de variables predictivas

\begin{tabular}{lcccc}
\hline Variables & Bosque nativo & Ríos & Tierras agropecuarias & Áreas antrópicas \\
\hline Bosque nativo & 1 & -0.032 & -0.107 & -0.298 \\
Ríos & -0.032 & 1 & -0.131 & -0.039 \\
Tierras agropecuarias & -0.107 & -0.131 & 1 & 0.246 \\
Áreas antrópicas & -0.298 & -0.039 & 0.245 & 1 \\
\hline
\end{tabular}

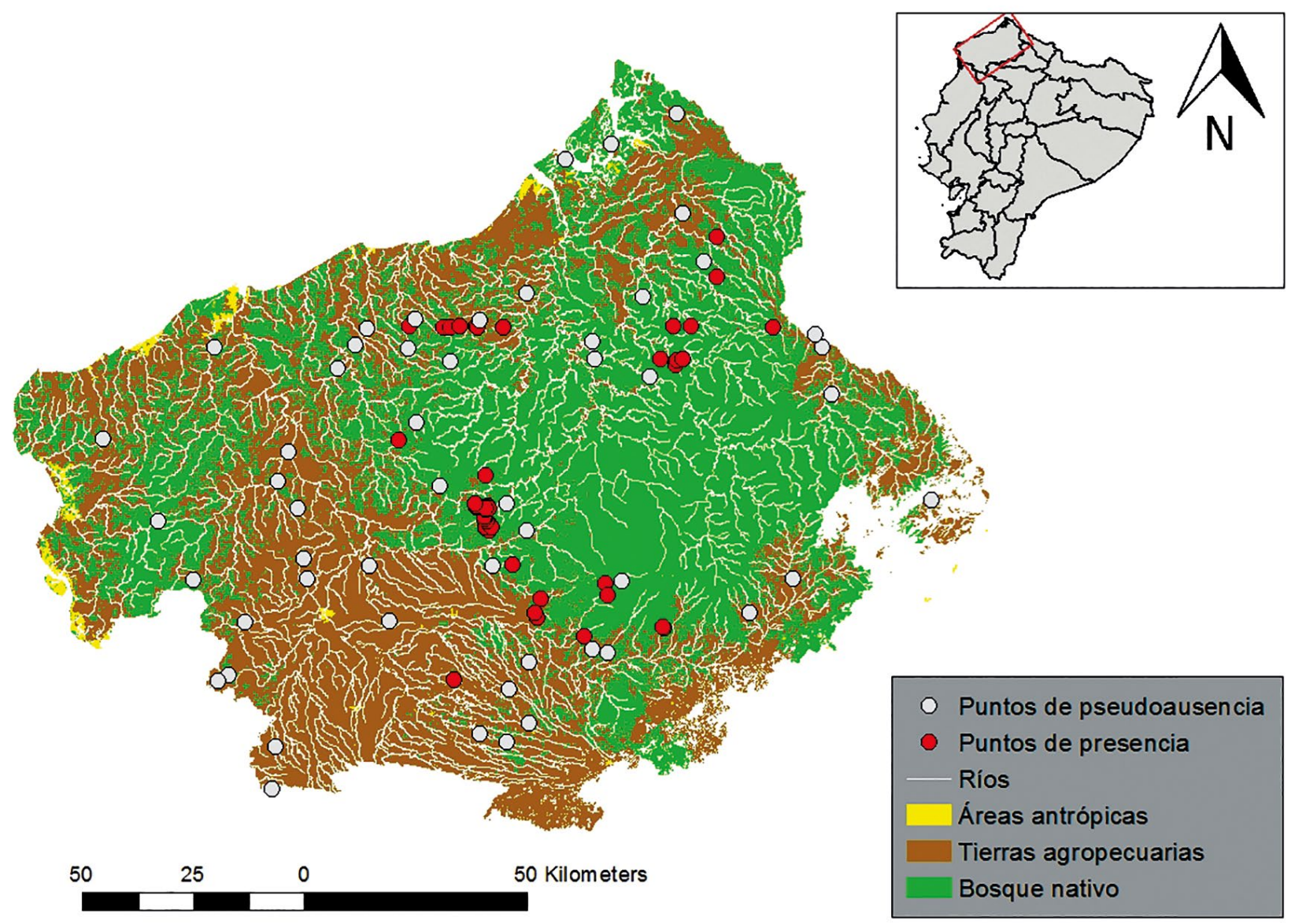

Figura 1. Localización de puntos de presencia y pseudoausencia en el área de estudio 


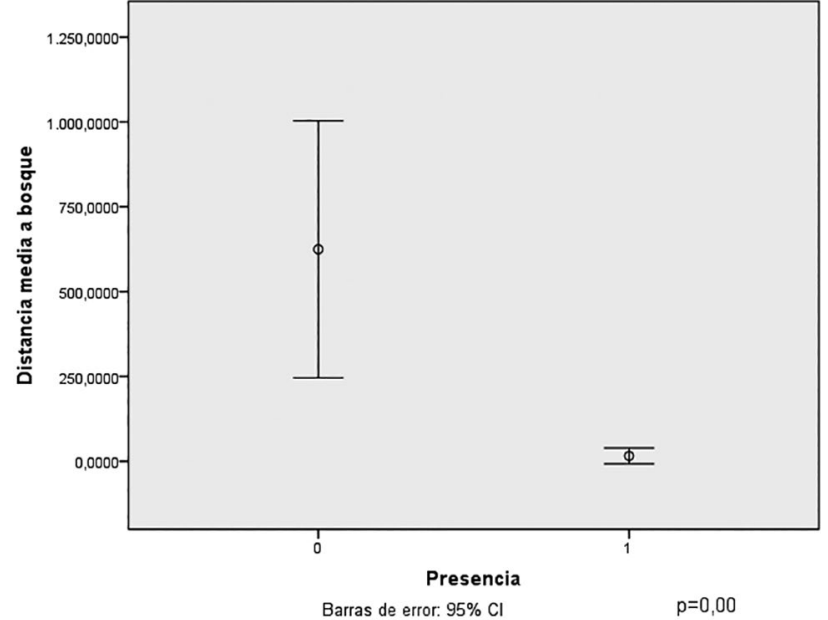

Figura 2. Distancia media desde los puntos de pseudoausencia (0) y puntos de presencia (1) a la categoría bosque nativo.

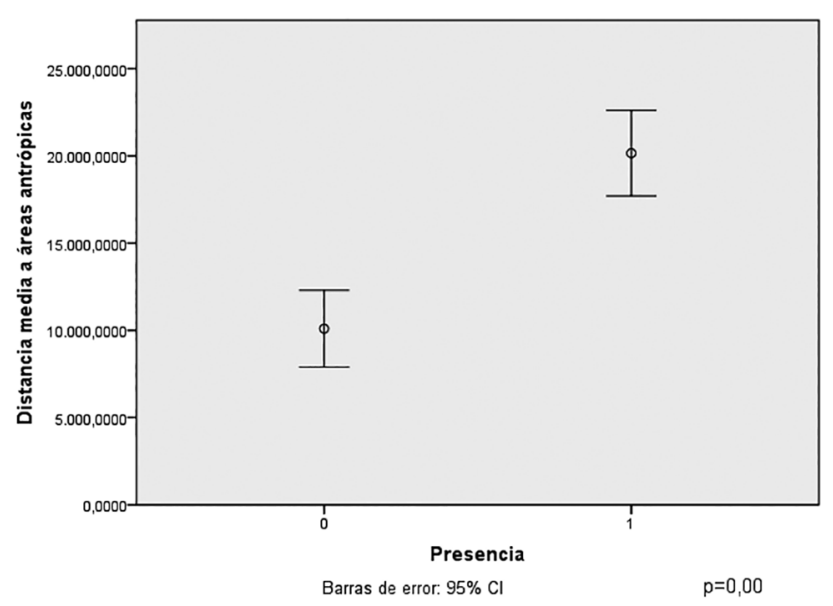

Figura 4. Distancia media desde los puntos de pseudoausencia (0) y puntos de presencia (1) a la categoría áreas antrópicas.

Influencia de la proximidad de variables predictivas en los patrones de presencia de Ateles fusciceps. - Se observó que los puntos de presencia de Ateles fusciceps están más cercanos a la categoría bosque nativo que los puntos de pseudoausencia. Se constata que no existe solapamiento de los intervalos de confianza de presencia y pseudoausencia y que la diferencia de la media de ambas distancias fue altamente significativa $(p=0.00)$, lo cual muestra que existe un patrón de presencia que evidentemente se relaciona con bosque nativo (Fig. 2).

Con respecto a la categoría ríos, se observó que los puntos de presencia estuvieron más cercanos que los puntos de pseudoausencia. Aunque existe un solapamiento parcial de los intervalos de confianza, la diferencia entre las medias de las distancias a los sitios de presencia y pseudoausencia fue marginalmente significativa $(p=0.05)$ (Figura 3 ).

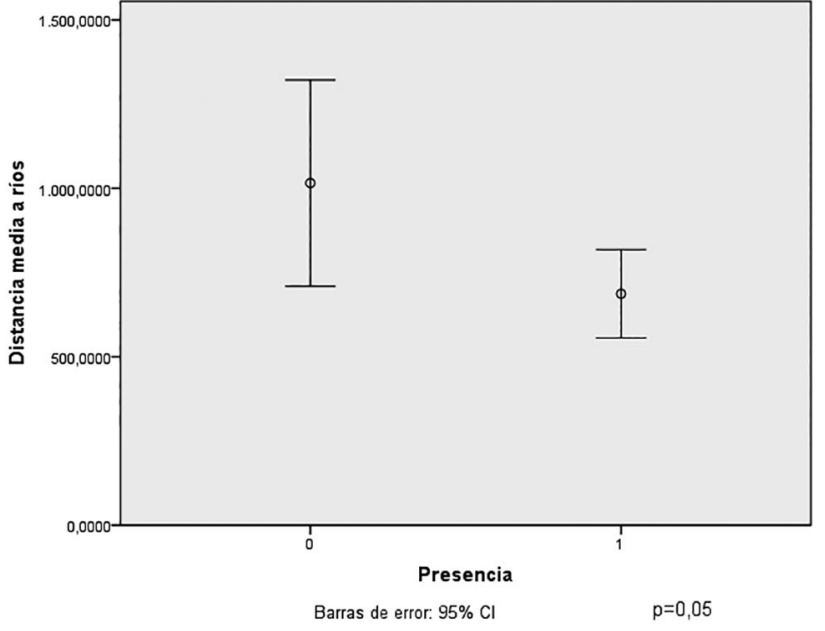

Figura 3. Distancia media desde los puntos de pseudoausencia (0) y puntos de presencia (1) a la categoría ríos.

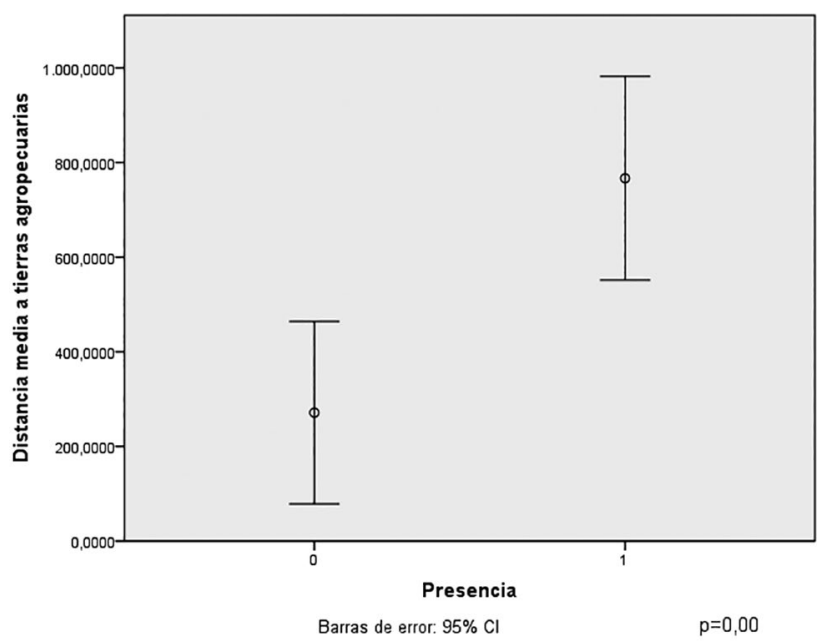

Figura 5. Distancia media desde los puntos de pseudoausencia (0) y puntos de presencia (1) a la categoría tierras agropecuarias.

En lo que se refiere a la categoría de áreas antrópicas, se observa que los puntos de presencia están más alejados que los puntos de pseudoausencia. Además, se constata que no existe solapamiento de los intervalos de confianza y que la diferencia entre las medias de las distancias a los sitios de presencia y pseudoausencia fue altamente significativa $(p=0.00)$ (Fig. 4). Este resultado muestra que los individuos de esta especie prefieren mantenerse alejados de zonas antrópicas, lo cual se constató durante el trabajo de campo pues no fueron observados en zonas cercanas a asentamientos humanos.

Finalmente, con respecto a la categoría tierras agropecuarias se observa que los puntos de presencia del primate están más alejados que los puntos de pseudoausencia. No se observa solapamiento de los intervalos de confianza de presencia y pseudoausencia y la diferencia entre las medias de ambas distancias es altamente significativa ( $p=0.00$ ) (Fig. 5). Este resultado muestra 
que los primates de esta especie prefieren mantenerse alejados de tierras agropecuarias.

Relación entre los patrones de presencia de Ateles fusciceps con atributos paisajísticos: Se observan dos modelos con los valores más altos de $\mathrm{AIC}_{\mathrm{w}}$ (Tabla 2). El modelo $1\left(\mathrm{AIC}_{\mathrm{w}}=0.48\right)$, incluyó tres variables: bosque nativo, ríos y zonas antrópicas. Este resultado, complementado con los análisis de proximidad, muestra que el primate prefiere sitios cercanos a bosque nativo, cercanos a ríos y alejados de áreas antrópicas. Mientras que el modelo $2\left(\mathrm{AIC}_{\mathrm{w}}=0.34\right)$, incluyó cuatro variables: bosque nativo, ríos, tierras agropecuarias, zonas antrópicas. Este resultado, complementado con los análisis de proximidad muestra que la preferencia del primate es hacia sitios cercanos a bosque nativo, cercanos a ríos, alejados de áreas antrópicas y alejados de tierras agropecuarias. Por otra parte, se observa que las variables más importantes son: bosque nativo (0.997), zonas antrópicas (0.989) y ríos (0.83), mientras que la variable menos importante es tierras agropecuarias $(0.438)$ (Tabla 3$)$.

Tabla 2. Selección del mejor modelo para predecir los patrones de presencia de Ateles fusciceps basado en Regresiones logísticas usando el Criterio de Información de Akaike $(A I C) . K=$ Número de parámetros, $\triangle A I C=$ Diferencia entre el $A I C$ del modelo y el menor AIC de todos los modelos, AICW = Peso del modelo.

\begin{tabular}{llcccc}
\hline$\#$ & Modelos & AIC & K & \multicolumn{1}{c}{$\Delta$ AIC } & AICw \\
\hline 1 & bosque nativo + ríos + zonas antrópicas & 100.847 & 4 & 0 & 0.4759 \\
2 & bosque nativo + ríos + tierras agropecuarias + zonas antrópicas & 101.495 & 5 & 0.648 & 0.3442 \\
3 & bosque nativo + tierras agropecuarias + zonas antrópicas & 104.264 & 4 & 3.417 & 0.0862 \\
4 & bosque nativo + zonas antrópicas & 104.396 & 3 & 3.549 & 0.0807 \\
5 & bosque nativo + ríos + tierras agropecuarias & 109.848 & 4 & 9.001 & 0.0053 \\
6 & bosque nativo + ríos & 110.475 & 3 & 9.628 & 0.0039 \\
7 & ríos + tierras agropecuarias + zonas antrópicas & 112.744 & 4 & 11.897 & 0.0012 \\
8 & bosque nativo + tierras agropecuarias & 113.241 & 3 & 12.394 & 0.0009 \\
9 & tierras agropecuarias + zonas antrópicas & 113.953 & 3 & 13.106 & 0.0007 \\
10 & bosque nativo & 115.137 & 2 & 14.29 & 0.0004 \\
11 & ríos + zonas antrópicas & 115.615 & 3 & 14.768 & 0.0003 \\
12 & zonas antrópicas & 117.696 & 2 & 16.849 & 0.0001 \\
13 & ríos + tierras agropecuarias & 134.239 & 3 & 33.392 & $2.670 \mathrm{E}-08$ \\
14 & tierras agropecuarias & 135.076 & 2 & 34.229 & $1.757 \mathrm{E}-08$ \\
15 & ríos & 144.05 & 2 & 43.203 & $1.977 \mathrm{E}-10$ \\
\hline
\end{tabular}

Tabla 3. Identificación de las variables más importantes para predecir la presencia de Ateles fusciceps.

\begin{tabular}{|c|c|c|c|c|}
\hline Modelos/Variables & Bosque nativo & Ríos & $\begin{array}{c}\text { Tierras } \\
\text { agropecuarias }\end{array}$ & $\begin{array}{c}\begin{array}{c}\text { Áreas } \\
\text { antrópicas }\end{array} \\
\end{array}$ \\
\hline bosque nativo & 0.0004 & & & \\
\hline bosque nativo + ríos + zonas antrópicas & 0.4759 & 0.4759 & & 0.4759 \\
\hline bosque nativo + ríos + tierras agropecuarias + zonas antrópicas & 0.3442 & 0.3442 & 0.3442 & 0.3442 \\
\hline bosque nativo + tierras agropecuarias + zonas antrópicas & 0.0862 & & 0.0862 & 0.0862 \\
\hline bosque nativo + zonas antrópicas & 0.0807 & & & 0.0807 \\
\hline tierras agropecuarias & & & $1.757 \mathrm{E}-08$ & \\
\hline bosque nativo + ríos + tierras agropecuarias & 0.0053 & 0.0053 & 0.0053 & \\
\hline bosque nativo + ríos & 0.0039 & 0.0039 & & \\
\hline ríos + tierras agropecuarias + zonas antrópicas & & 0.0012 & 0.0012 & 0.0012 \\
\hline bosque nativo + tierras agropecuarias & 0.0009 & & 0.0009 & \\
\hline tierras agropecuarias + zonas antrópicas & & & 0.0007 & 0.0007 \\
\hline ríos + zonas antrópicas & & 0.0003 & & 0.0003 \\
\hline zonas antrópicas & & & & 0.0001 \\
\hline ríos + tierras agropecuarias & & $2.670 \mathrm{E}-08$ & $2.670 \mathrm{E}-08$ & \\
\hline ríos & & $1.977 \mathrm{E}-10$ & & \\
\hline TOTAL & 0.9977 & 0.8309 & 0.4387 & 0.9896 \\
\hline
\end{tabular}




\section{Discusión}

Los resultados obtenidos sugieren que los esfuerzos de conservación de $A$. fusciceps deben enfocarse en áreas que tengan bosque nativo y cuerpos de agua, y que estén alejadas de tierras agrícolas y asentamientos humanos. La asociación con el bosque nativo fue evidente durante los recorridos en el campo, pues todos los individuos fueron encontrados únicamente en estas áreas, entendiéndose que la tala de bosque es un factor que afecta directamente su presencia por la destrucción de hábitat.

Muchas de las especies de árboles presentes en el bosque nativo son preferidas por $A$. fusciceps para su alimentación, pero también son muy cotizadas en el comercio de madera, como el sande (Brosimum utile), que presenta los promedios más altos en el tiempo dedicado a la alimentación para el mono araña (Moscoso 2010, Tapia 2014). Además, el bosque nativo, por poseer abundantes árboles de grandes diámetros, permite la continuidad en la cobertura del dosel, siendo importante para el desplazamiento de $A$. fusciceps (Estrada et al. 1994).

Una opción efectiva para evitar la destrucción del bosque nativo es la compra de tierras. Esta medida requiere de gran cantidad de fondos, sin embargo, en la actualidad sucede ggracias a la intervención de la Reserva Tesoro Escondido, quienes actúan de tal manera en la Comunidad Tesoro Escondido con el fin de conservar al mono araña de cabeza café. Es trascendental que proyectos similares se efectúen en otros lugares donde habita este primate, pero sería importante que como complemento se disponga de fondos para trabajar con gente de comunidades cercanas mediante talleres de educación ambiental en temas de reforestación, ofreciéndoles fuentes de trabajo que se enfoquen en actividades de conservación de bosques.

Youlatos (2002), menciona que los monos araña (Ateles spp.) son exclusivamente arborícolas y pasan la mayoría del tiempo en doseles altos, sin embargo, de acuerdo a los resultados de este estudio, se encontró que Ateles fusciceps prefiere zonas de bosque cercanas a ríos, esto es novedoso, pues no ha sido reportado en estudios previos. Conociendo que las especies de Ateles raramente bajan al suelo (Campbell et al. 2005), se esperaría que su presencia no esté relacionada con ríos, sin embargo, en el sur de México, Ateles geoffroyi y Alouatta pigra se desplazan hacia sitios cercanos a ríos y los cruzan (Chaves \& Stoner 2010). Asimismo, se observó que las poblaciones de Ateles de Sudamérica descendían al suelo para beber agua de riachuelos e ingerir tierra (Campbell et al. 2005). En Colombia se observó a grandes subgrupos del mono araña bebiendo agua en saladeros ubicados cerca a orillas de ríos (Izawa et al. 1975, Izawa 1979), por lo que sería probable que el mismo fenómeno esté ocurriendo en el noroccidente ecuatoriano con $A$. fusciceps, posiblemente, además de beber agua en estos sitios, también ingieren tierra para obtener minerales (Dew 2005). Asimismo, de acuerdo a la versión de un habitante de la comunidad Tesoro Escondido, se ha observado a este primate en las orillas de ríos. Al no existir estudios anteriores con esta especie que presenten resultados similares, sería muy importante que nuevas investigaciones consideren profundizar análisis en este aspecto, de esta manera se podría tener mayor cantidad de fuentes de información para proponer medidas adecuadas en pro de la conservación de este primate.

Adicionalmente, se evidencia que los requerimientos de hábitat para Ateles fusciceps se enfocan en áreas que no presenten intervención humana, resultado concordante con Moscoso (2010), quien menciona que esta especie evita lugares con cualquier forma de intervención humana. Se deduce que la expansión de la población humana hacia zonas de bosque donde habita este primate, afecta directamente en sus patrones de presencia. Es así, que factores como la tala de bosques, la cacería, la expansión de la frontera agrícola, entre otras, estarían relacionados con el hecho de que $A$. fusciceps se encuentre entre los 25 primates más amenazados del mundo (Mittermeier et al. 2012). Se entiende que el establecimiento de asentamientos humanos propicia actividades como la cacería y la destrucción de bosques, lo que disminuye su abundancia, disponibilidad de hábitat y cantidad de recursos alimenticios, motivos por los que se explicaría que $A$. fusciceps prefiera estar lejos de este tipo de áreas. Precisamente esta especie es preferida para la cacería sobre otras como Alouatta palliata y Cebus capucinus debido a la palatabilidad de su carne. La cacería es una práctica regular para las comunidades indígenas Chachis, por lo que estar cerca de áreas antrópicas les hace más vulnerables a esta actividad (Madden \& Albuja 1989).

En lo que concierne a tierras agrícolas, se conoce que la conversión de tierras para la agricultura en el noroccidente ecuatoriano ha conllevado a que se pierda el $80 \%$ del hábitat de esta especie, lo cual implicaría altos riesgos de extinción (Baird, 2007; Spaan et al. 2013). Un ejemplo claro es el establecimiento de grandes áreas de monocultivo de palma aceitera en Esmeraldas, la cual se cultiva con el fin de extraer aceite de sus frutos para la producción de aceite de cocina, jabón, producción de biocombustibles, entre otros (Sanborn \& Delgado 2006). Obviamente esta actividad implica la deforestación de grandes áreas de bosque, poniendo en riesgo la supervivencia de este primate.

A pesar de los grandes perjuicios que supone la expansión de la frontera agrícola, Cervera \& Griffith (2016) reportaron la presencia de Ateles fusciceps en la provincia de Manabí, en un paisaje deforestado en un $89 \%$, compuesto por pastos para ganado, cultivos de cacao, plantaciones de árboles y remanentes de bosque húmedo tropical. Esto da una pauta para que entre las medidas para la conservación de este primate se considere la implementación de sistemas de agroforestería, lo cual consiste en la creación de corredores biológicos con vegetación nativa a lo largo de campos agrícolas y que conecten fragmentos de bosque, esto podría ayudar al movimiento de la especie a lo largo del paisaje y de cierta manera mitigar los efectos de la agricultura (Boyle \& Smith 2010). De esta forma, tanto la gente como el primate serían beneficiados, pues las comunidades que habitan alrededor, ven a la agricultura como un medio de subsistencia. Así, se estaría promoviendo el equilibrio entre las necesidades de la gente para subsistir y del primate para sobrevivir.

Es importante el desarrollo de políticas que permitan la conservación de $A$. fusciceps sin descuidar el bienestar de las comunidades, siendo importante su involucramiento en las acciones de conservación que se lleven a cabo. Seguramente de esta manera se podrá establecer una base importante para planificar la delimitación de áreas protegidas, siendo trascendental que para este caso se consideren sectores que posean bosque nativo y cuerpos de agua y que estén alejados de asentamientos humanos y tierras agropecuarias. 


\section{Agradecimientos}

Agradezco a Guillermo Medrano y Luisa Vizcaíno por su colaboración intelectual y económica, factores que fueron fundamentales para que este trabajo sea realizado. A Tali Morelos y Galo Conde por su predisposición y apertura para facilitar mi ingreso a Tesoro Escondido. A Iván Jácome, Mercedes Gavilanez y Paulina Guarderas, por sus sugerencias y comentarios, para la estructuración de este documento.

\section{Literatura citada}

Agetsuma N., \& N. Noma. 1995. Rapid shifting of foraging pattern by Yakushima macaques (Macaca fuscata yakui) in response to heavy fruiting of Myricarubra. International Journal of Primatology 16: 247-260. doi: https://doi.org/10.1007/ BF02735480

Akaike H. 1992. Information theory and an extension of the maximum likelihood principle. En Kotz, S. y N. Johnson. (eds) Breakthr. stat., 1: 610-624. Springer-Verlag, Londres, Reino Unido. doi: https://doi.org/10.1007/978-1-46120919-5_38

Altmann S. \& J. Altmann. 1970. Baboon Ecology. Chicago: University of Chicago Press.

Andrén H. 1994. Effects of habitat fragmentation on birds and mammals in landscapes with differents proportions of suitable habitat: A review. Oikos, 71, 355-366. Doi: https://doi. org/10.2307/3545823

Baird A. 2007. RAPID - Development of playback for rapid population assessment of the critically endangered brown-headed spider monkey (Ateles fusciceps) in Ecuador. Master thesis, Oxford Brookes University. England.

Boada C. 2014. Ateles fusciceps. Citado en: Santiago Burneo (ed). Mamíferos de Ecuador. Quito, Ecuador. [en línea]. Versión 2013.0. Museo de Zoología, Pontificia Universidad Católica del Ecuador. Quito. (Disponible en: http://zoologia.puce.edu.ec/vertebrados/mamiferos/FichaEspecie. aspx?Id=85. Consultado el: viernes, 20 de marzo de 2015).

Boyle S. \& A. Smith. 2010. Can landscape and species characteristics predict primate presence in forest fragments in the Brazilian Amazon?. Biological Conservation, 5, 1134-1143. doi: https://doi.org/10.1016/j.biocon.2010.02.008

Burnham K., \& D. Anderson. 2002. Model selection and multimodel inference: a practical information-theoretic approach. Second edition. Springer-Verlag, New York, USA.

Campbell C., F. Aureli, C. Chapman, G. Ramos-Fernández, K. Matthews, S. Russo, S. Suarez, \& L. Vick, 2005. Terrestrial behavior of Ateles spp. International Journal of Primatology, 26, 1039-1051. doi: https://doi.org/10.1007/ s10764-005-6457-1

Cervera L. \& D. Griffith, 2016. New population and range extension of the Critically Endangered Ecuadorian brown-headed spider monkey (Ateles fusciceps fusciceps) in western Ecuador. Tropical Conservation Science, 9, 167-177. doi: https://doi.org/10.1177/194008291600900109

Chaves O., \& K. Stoner. 2010. River crossings by Ateles geoffroyi and Alouatta pigra in southern Mexico: A preliminary report. Revista Chilena de Historia Natural, 83, 435-442. Doi: https://doi.org/10.4067/S0716-078X2010000300010

Cowlishaw G. 1997. Trade-offs between foraging and predation risk determine habitat use in a desert baboon population. Animal Behaviour, 53, 667-686. Doi: https://doi.org/10.1006/ anbe. 1996.0298

Cuarón A., A. Morales, A. Shedden, E. Rodríguez-Luna \& P. de Grammont. 2008. Ateles fusciceps ssp. fusciceps. Citado en: IUCN 2013. IUCN Red List of Threatened Species. Version 2013.2. <www.iucnredlist.org>.

Cueva X. 2008. Aspectos demográficos de Alouatta palliata aequatorialis y Ateles fusciceps fusciceps en el noroccidente ecuatoriano. Trabajo de Grado presentado como requisito parcial para optar al Título de Licenciado en Ciencias Biológicas y Ambientales. Universidad Central del Ecuador. Quito.
Defler T. 1996. Aspects of the ranging pattern in a group of wild woolly monkeys (Lagothrix lagotricha). American Journal of Primatology, 38, 289-302. Doi: https://doi. org/10.1002/(SICI) 1098-2345(1996)38:4<289::AIDAJP1>3.0.CO;2-V

Dew J. 2005. Foraging, Food Choice, and Food Processing by Sympatric Ripe-Fruit Specialists: Lagothrix lagotricha poeppiggi and Ateles belzebuth belzebuth. International Journal of Primatology, 26, 1123-1129. Doi: https://doi. org/10.1007/s10764-005-6461-5

Dodson C., \& A. Gentry. 1993. Extinción Biológica en el Ecuador occidental. Extinción Biológica en el Ecuador occidental. Pp. 27-57. Citado en: Mena, P \& Suárez, L. (Ed.). La investigación para la Conservación de la Diversidad Biológica en el Ecuador. EcoCiencia. Quito.

Estrada A., R. Coates-Estrada, \& D. Meritt. 1994. Non flying mammals and landscape changes in the tropical rain forest region of Los Tuxtlas, Mexico. Ecography, 17: 229-241. Doi: https://doi.org/10.1111/j.1600-0587.1994.tb00098.x

Fahrig L. 2003. Effects of habitat fragmentation on biodiversity. Annual review of ecology evolution and systematics, 34, 487-515. Doi: https://doi.org/10.1146/annurev.ecolsys.34.011802.132419

Forman, R., \& M. Godron, 1986. Landscape Ecology, 619 pp. Jhon Wiley \& Sons, New York.

IGM (Instituto Geográfico Militar). (2013). Base continua, escala $1: 250.000$. Quito, Ecuador.

Izawa K. 1975. Foods and feeding behavior of monkeys in the Upper Amazon Basin. Primates 16: 295-316. Doi: https://doi. org/10.1007/BF02381557

Izawa K., K. Kimura, \& A. Samper Nieto. 1979. Grouping of the wild spider monkey. Primates, 20, 503-512. Doi: https:// doi.org/10.1007/BF02373432

MAE-MAGAP. (2015). Mapa de cobertura y uso de la tierra del Ecuador continental 2013 - 2014, escala 1:100.000.

Madden R., \& L. Albuja. 1989. Estado actual de Ateles fusciceps fusciceps en el noroccidente ecuatoriano. Revista Politécnica 14, 113-157.

Mazerolle M. 2006. Improving data analysis in herpetology: using Akaike's Information Criterion (AIC) to assess the strength of biological hypotheses. Amphibia-Reptilia, 27(2): 169180. Doi: https://doi.org/10.1163/156853806777239922

Mittermeier R., C. Valladares-Pâdua, A. Rylands, A. Eudley, M. Butynski, J. Ganzhorn, R. Kormos, J. Aguiar, \& S. Walker. 2005. Primates in peril: the world's 25 most endangered primates 2004-2006. Primate Conservation, 1-28.

Mittermeier R., C. Schwitzer, A. Rylands, L. Taylor, F. Chiozza, E. Williamson, \& J. Wallis, (eds.). 2012. Primates in Peril: The World's 25 Most Endangered Primates 2012-2014. IUCN/SSC Primate Specialist Group (PSG), International Primatological Society (IPS), Conservation International (CI), and Bristol Conservation and Science Foundation, Bristol, UK. Pp. 40.

Moscoso P. 2010. Estado poblacional del mono ara-a de cabeza café (Ateles fusciceps) en el noroccidente del Ecuador, con notas ecológicas de una relación interespecífica con Alouatta palliata. Trabajo de Grado presentado como requisito parcial para optar al Título de Licenciada en Ciencias Biológicas. Pontificia Universidad Católica del Ecuador. Quito.

Myers N., R. Mittermeier, C. Mittermeier, G. Da Fonseca, \& J. Kent. 2000. Biodiversity hotspots for conservation priorities. Nature, 403, 853-858. Doi: https://doi. org/10.1038/35002501

Olupot W., C. Chapman, P. Waser, \& G. Isabirye-Basuta. 1997. Mangabey (Cercocebus albigena) ranging patterns in relation to fruit availability and the risk of parasite infection in Kibale National Park, Uganda. American Journal of Primatology, 43, 65-78. Doi: https://doi.org/10.1002/ (SICI) 1098-2345(1997)43:1<65::AID-AJP5>3.0.CO;2-W

Risser P., J. Karr, \& R. Forman, R. 1984. Landscape Ecology: Directions and Approaches. Special Publication, 2, 11.

Sanborn C., \& A. Delgado. 2006. Palmas del Espino: Harvesting hope in the upper Huallaga. Revista Harvard Review of Latin America 6:6-9. 
Saunders D., R. Hobbs, \& C. Margules. 1991. Biological consecuences of ecosystem fragmentation: a review. Conservation Biology, 5, 18-32. doi: https://doi. org/10.1111/j.1523-1739.1991.tb00384.x

Spaan D., C. Morelos-Juárez, M. Peck, \& V. Nijman. 2013. Factors affecting the presence of the critically endangered Brownheaded spider monkey (Ateles fusciceps fusciceps) in NW Ecuador. Folia Primatológica, 84, 239-346.

SPSS. 2009. Statistical Packages for the Social Sciences 18.0. Chicago, IL: SPSS, Inc.

Tapia A. 2014. Estudio piloto sobre la ecología alimentaria del Mono Araña de Cabeza Marrón (Ateles fusciceps) en el Chocó Ecuatoriano. Trabajo de Grado presentado como requisito parcial para optar al Título de Licenciada en Biología. Universidad San Francisco de Quito. Ecuador.
Turner M. 2005. Landscape ecology: what is the state of the science?. Annual review of ecology evolution and systematics, 36, 319-344. doi: https://doi.org/10.1146/annurev.ecolsys.36.102003.152614

Youlatos D. 2002. Positional behavior of black spider monkeys (Ateles paniscus) in French Guiana. International Journal of Primatology, 23, 1071-1093. doi: https://doi. org/10.1023/A:1019602116805

Zhang S., \& L. Wang. 1995. Consumption and seed dispersal of Ziziphus cinnamomum (Rhamnaceae) by two sympatric primates (Cebus apella and Ateles paniscus) in French Guiana. Biotropica, 27, 397-401. doi: https://doi. org/10.2307/2388926 